\title{
Does inflammatory bowel disease increase the risk of lower urinary tract tumors: a meta-analysis
}

\author{
Chi Zhang", Shengzhuo Liu", Liao Peng, Jiapei Wu, Xiao Zeng, Yiping Lu, Hong Shen, Deyi Luo^ \\ Department of Urology, Institute of Urology, West China Hospital, Sichuan University, Chengdu, China \\ Contributions: (I) Conception and design: D Luo, Y Lu, H Shen, C Zhang, S Liu; (II) Administrative support: X Zeng, Y Lu, H Shen, D Luo; (III) \\ Provision of study materials or patients: None; (IV) Collection and assembly of data: C Zhang, S Liu, L Peng, J Wu, X Zeng; (V) Data analysis and \\ interpretation: C Zhang, S Liu, L Peng, J Wu; (VI) Manuscript writing: All authors; (VII) Final approval of manuscript: All authors. \\ "These authors contributed equally to this work. \\ Correspondence to: Deyi Luo, PhD. Department of Urology, West China Hospital, Sichuan University, Wuhou District, Chengdu 610000, China. \\ Email: luodeyi1985@163.com.
}

Background: Inflammatory bowel disease, including ulcerative colitis and Crohn's disease, is characterized by chronic inflammation that could be a risk factor for extraintestinal cancer. The aim of this study is to evaluate whether inflammatory bowel disease is related to the risk of lower urinary tract tumors.

Methods: A systematical research was performed on various medical databases including PubMed, the Cochrane Library, Embase and Web of Science from inception to April 2020. Data were independently extracted by two reviewers. The Newcastle-Ottawa Scale and the Oxford Centre for Evidence-Based Medicine criteria were used to assess the quality of included articles. The analysis was completed by STATA version 14.2.

Results: Six hundred and twelve of records were initially identified and 16 studies were included in the final analysis. In general, inflammatory bowel disease patients were not at increased risk of prostate cancer, bladder cancer and male genital cancer. In the subgroup analysis, Crohn's disease patients seemed to have borderline increased risk of prostate cancer [standardized incidence ratio: 1.05; 95\% confidence interval (CI): $\left.0.90-1.21 ; \mathrm{I}^{2}=15.1 \%\right]$ and bladder cancer (standardized incidence ratio: $1.19 ; 95 \%$ CI: $0.94-1.44 ; \mathrm{I}^{2}=0.0 \%$ ), and ulcerative colitis patients seemed to have borderline increased risk of prostate cancer (standardized incidence ratio: 1.13 ; $95 \% \mathrm{CI}$ : $\left.0.93-1.33 ; \mathrm{I}^{2}=73.5 \%\right)$.

Conclusions: Inflammatory bowel disease did not significantly increase the risk of prostate cancer, bladder cancer and male genital cancer. Crohn's disease patients seemed to have a higher risk of prostate cancer and bladder cancer, and ulcerative colitis patients seemed to have a higher risk of prostate cancer. ulcerative colitis patients in East Asian countries have significantly increased prostate cancer risk.

Keywords: Inflammatory bowel disease (IBD); Crohn's disease; prostate cancer (PCa); bladder cancer (BCa); male genital cancer (MGCa)

Submitted Jun 20, 2020. Accepted for publication Oct 16, 2020.

doi: $10.21037 /$ tau-20-1020

View this article at: http://dx.doi.org/10.21037/tau-20-1020

^ ORCID: 0000-0002-9436-036X. 


\section{Introduction}

Inflammatory bowel disease (IBD), comprising ulcerative colitis (UC) and Crohn's disease (CD), is characterized by chronic gastrointestinal tract inflammation. In the past decades, a significantly increased incidence of IBD has been observed worldwide, especially in eastern countries (1). IBD has been a great health burden to the society for its high morbidity and tight association with gastrointestinal malignancy $(2-4)$, as well as numerous extraintestinal malignancies $(5,6)$.

Though mechanism underlying IBD and oncogenesis has not been clearly elucidated, it was reported that inflammation not only serves as host's response to malignant tumor but also elicit carcinogenesis $(7,8)$. Moreover, immunosuppressant medications, which are widely used for IBD treatment, are believed to be responsible for elevated risk of cancers like non-Hodgkin lymphoma, acute myeloid leukemia, non-melanoma skin cancers and urinary tract cancers (9-12). From these knowledges, a concept about the interplay between IBD and malignancy could be established, which depicts the inflammation induced immune dysregulation and immunosuppressant medications use as key factors in IBD related malignancies. However, this indirect evidence-based hypothesis may not apply to all kinds of malignant tumors, especially extraintestinal tumors. Direct evidence from studies evaluating the association between certain malignancy and IBD could be of great value in prognosis predicting and providing guidance for cancer surveillance. To our knowledge, no meta-analysis has been performed to evaluating the risk of bladder cancer $(\mathrm{BCa})$ or male genital cancer (MGCa) in IBD patients. A recent study reported an increased risk of prostate cancer (PCa) in IBD patients (13). With the aim of evaluating the risk of lower urinary tract cancers including $\mathrm{PCa}, \mathrm{BCa}$ and MGCa in IBD patients, we performed this meta-analysis. We presented the following article in accordance with the PRISMA reporting checklist (available at http://dx.doi. org/10.21037/tau-20-1020).

\section{Methods}

\section{Search strategy}

We conducted this meta-analysis according to the guideline of Preferred Reporting Items for Systematic Reviews and Meta-Analyses (PRISMA) Statement (14). Electronic databases including PubMed, the Cochrane Library,
Embase and Web of Science was initially searched from inception to April 2020 with no language limitation. We used the following Medical Subject Heading (MeSH) terms and related synonyms: "Inflammatory Bowel Diseases", "Urinary Bladder Neoplasms", "Prostatic Neoplasms", and "Genital Neoplasms, Male". The complete search strategy was described in the Supplementary file. We scrutinized all potentially eligible studies, regardless of the primary outcomes or language. Besides, references from related articles were also retrieved manually to ensure a comprehensive search.

\section{Study selection}

Studies were regarded as eligible if they satisfied the following inclusion criteria: (I) patients developed with $\mathrm{BCa}$, $\mathrm{PCa}$, or MGCa after being diagnosed with IBD, irrespective of UC or CD; (II) standardized incidence ratio (SIR) or relative risk (RR) with $95 \%$ confidence intervals $(\mathrm{CIs})$ were used to evaluate the association between IBD and risk of lower urinary tract tumors (BCa, PCa or MGCa); (III) population-based cohort studies or case-control studies; (IV) the follow-up of included studies should be more than 1 year. If the study population overlapped, we used the most recently published or most informative literature. Exclusion criteria were as follows: (I) any study which did not meet the inclusion criteria; (II) meeting abstracts, review or meta-analysis; (III) data not available. Figure 1 sketches the PRISMA flow diagram of this meta-analysis.

\section{Data extraction and quality assessment}

Two independent authors screened the search results based on title, abstract, and, finally, full text. Disagreements were resolved by discussion or a third party. We prospectively developed a homogeneous form to extract data. Data were independently extracted by two reviewers. The following data were extracted: the first author's name, year of publication, country, study design, period, sample size, cancer types, outcomes (SIRs or RRs).

Two authors independently evaluated the methodological bias of included studies through the Newcastle-Ottawa Scale (NOS) (15). The NOS used a "star system" to assess the quality of study from three perspectives: the selection of the studies, the comparability of studies, and the assessment of outcome. Whenever seven or more of nine stars were received, the study was considered as high quality. 

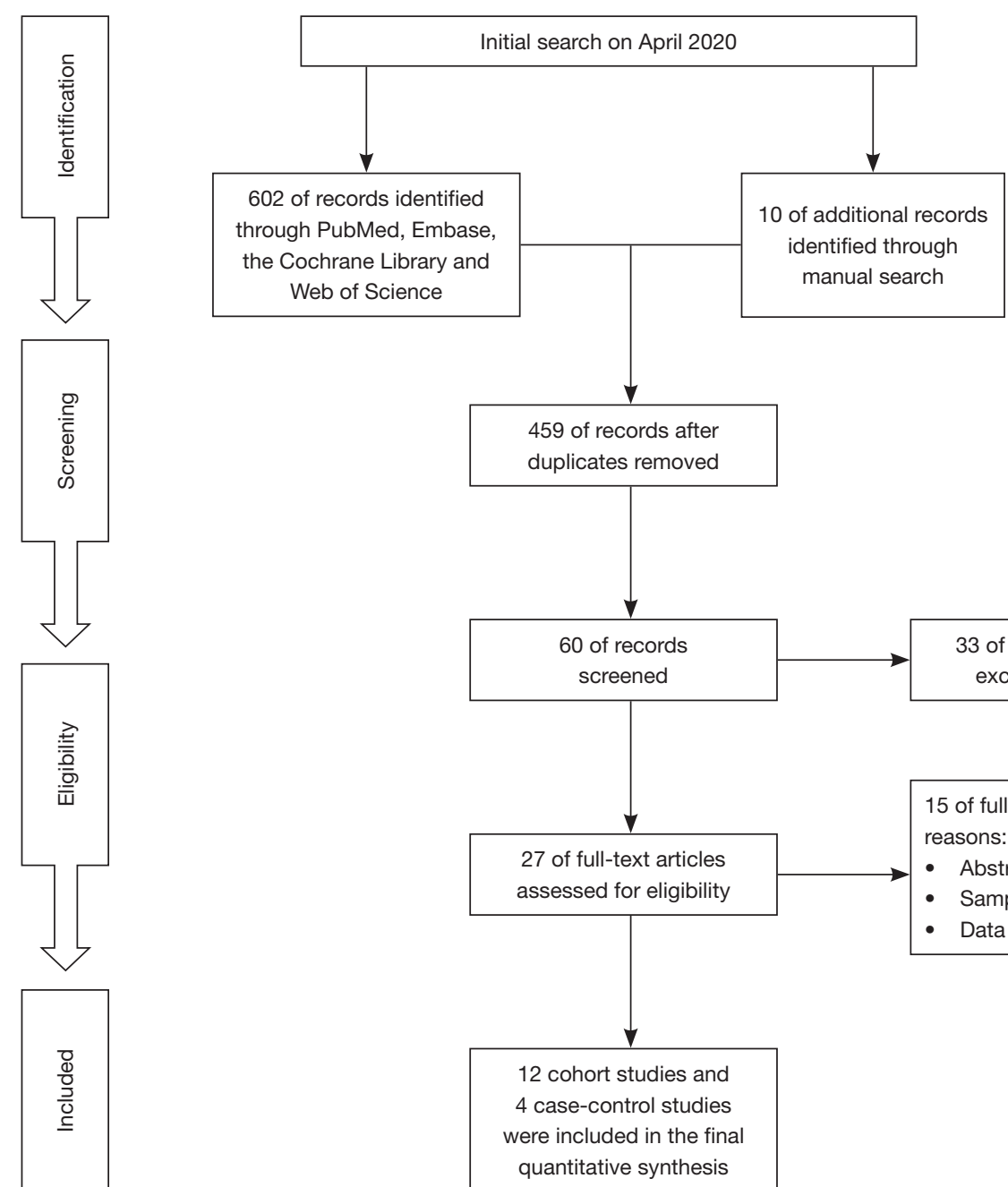

459 of records after duplicates removed
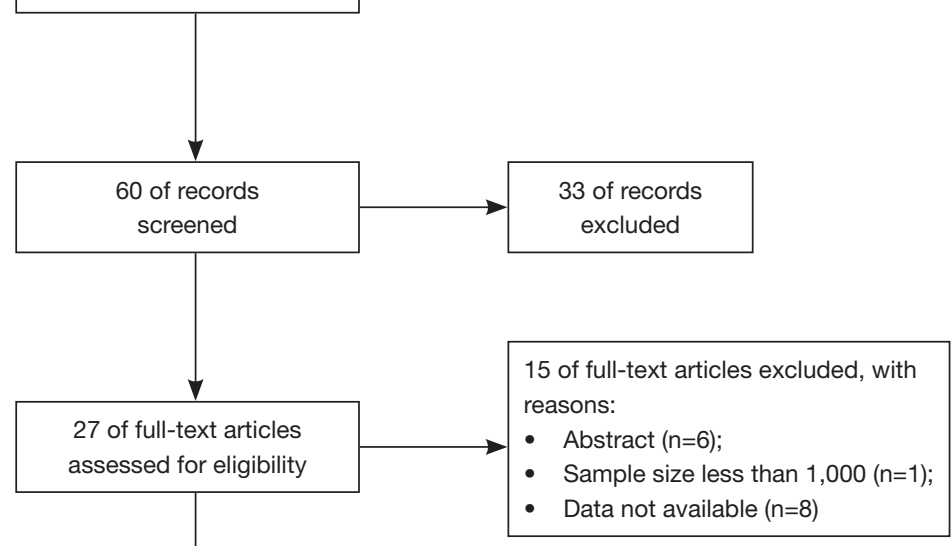

Figure 1 The PRISMA flowchart showing the study selection process of this meta-analysis.

Moreover, two reviewers independently rated the level of evidence of the included articles using the Oxford Centre for Evidence-Based Medicine criteria (16); This scale graded studies from strongest (level 1) to weakest (level 5) strength of evidence on the basis of study design and data quality.

\section{Statistical analysis}

Data were expressed as SIR or RR with $95 \%$ CIs. The $\mathrm{Q}$ and $\mathrm{I}^{2}$ statistics were calculated to estimate the heterogeneity among studies. If $\mathrm{P}>0.1$ or $\mathrm{I}^{2} \leq 50 \%$, we used the fixed-effect models. Otherwise, a random-effect model was used. Besides, we reported the pooled analysis according to the cancer types and IBD subtype. Funnel plots were used to assess the possibility of publication bias, and the asymmetry of funnel plots was evaluated by Egger's test and Begg's test; We considered significant publication bias as a $\mathrm{p}$ value less than 0.1 . Furthermore, we conducted sensitivity analyses to evaluate the robustness of the pooled results. Statistical significance was set at $\mathrm{P}<0.05$. This metaanalysis was completed by STATA version 14.2 (StataCorp LP, College Station, TX, USA).

\section{Results}

\section{Search results}

Six hundred and twelve of records were initially identified 
through PubMed, Embase, the Cochrane Library and Web of Science from inception to April 2020, resulting 12 cohort studies (12,17-27) and 4 case-control (28-31) studies included in the final analysis based on title, abstract, and full text (Figure 1). A total of 511,259 participants were included in this population-based study. In the cohort studies, 10 of 12 studies with 174,094 patients reported available data for PCa, 9 of 12 studies with 136,502 patients were available for BCa and 6 of 12 studies were available for MGCa. In the case-control studies, a total of 4 studies containing 335,167 participants reported related outcomes of $\mathrm{PCa}$, and 2 of 4 studies with 285,534 participants were available for BCa. Table 1 details the main characteristics of included studies in this study.

\section{Meta-analysis results}

\section{PCa}

For cohort studies, the risk of PCa in patients with IBD were of borderline significant increase (SIR: 1.09; 95\% CI: $0.95-1.22 ; \mathrm{I}^{2}=61.2 \%$; Figure 2$)$ when compared with the background population, as was the risk of subgroup of UC (SIR: $1.13 ; 95 \%$ CI: $0.93-1.33 ; \mathrm{I}^{2}=73.5 \%$; Figure 2 ) and CD (SIR: 1.05 ; 95\% CI: $0.90-1.21 ; \mathrm{I}^{2}=15.1 \%$; Figure 2). For case-control studies, the results indicated that there was no significant difference between IBD group and IBDfree group regarding the risk of PCa (RR: $1.70 ; 95 \%$ CI: 0.87-3.33; Figure 2) with great between-study heterogeneity $\left(\mathrm{I}^{2}=93.5 \% ; \mathrm{P}=0.000 ;\right.$ Figure 2). Subgroup analysis revealed that IBD patients in Eastern countries have significantly increased PCa risk (SIR: 2.62; 95\% CI: 1.38-3.87; $\mathrm{I}^{2}=13.6 \%$; Figure 3), especially for UC patients (SIR: 3.01; 95\% CI: $1.75-4.27 ; \mathrm{I}^{2}=0.0 \%$; Figure 3).

\section{BCa}

For cohort studies, no significantly decreased risk of $\mathrm{BCa}$ was observed in patients with IBD (SIR: 0.99; 95\% CI: $0.87-1.12 ; \mathrm{I}^{2}=0.0 \%$; Figure 2 ) and UC subgroup (SIR: 0.92; 95\% CI: $0.77-1.06 ; \mathrm{I}^{2}=0.0 \%$; Figure 2), whereas a trend toward an increased risk of $\mathrm{BCa}$ was detected in patients with CD subgroup (SIR: 1.19; 95\% CI: 0.94-1.44; $\mathrm{I}^{2}=0.0 \%$; Figure 2). Only two case-control studies provided data on $\mathrm{BCa}$ in UC and CD combined, revealing that IBD patients seemed to have a higher risk of $\mathrm{BCa}$ than IBD-free patients (RR: 1.25 ; 95\% CI: $0.68-2.31 ; \mathrm{I}^{2}=37.5 \%$; Figure 2).

\section{MGCa}

Data from six cohort studies $(17,18,21,22,24,26)$ showed that IBD or IBD subtype (UC and CD) did not significantly increase the risk of MGCa. The pooled SIRs were 1.30, 1.20 and 1.29 for IBD, UC and CD, respectively (Figure 2).

\section{Publication bias and sensitivity analysis}

No significant asymmetry was observed in the present study in terms of $\mathrm{PCa}$ and $\mathrm{BCa}$ through the corresponding funnel plots (Figure 4). The P values of Egger's test for PCa, BCa and MGCa were 0.234, 0.194 and 0.276, respectively. Furthermore, sensitivity analysis revealed that there was no significant change of the pooled results for all outcomes observed through excluding each study from the metaanalysis sequentially (Figure 4).

\section{Discussion}

In this study, we found there was no significant difference between IBD patients and background population regarding the risk of $\mathrm{PCa}, \mathrm{BCa}$ or MGCa. However, a trend toward higher risk of $\mathrm{PCa}$ was suggested in patients with IBD, CD, and UC. For BCa, CD patients seemed to have a higher risk. As was previously reported, lower incidence rate of $\mathrm{PCa}$ was observed in East Asian countries when compared with that in other regions (32), we performed a subgroup analysis to evaluate the risk of IBD related PCa in East Asian countries. Different from the general results above, a significantly increased risk of $\mathrm{PCa}$ in East Asian IBD patients was detected (SIR: 2.62; 95\% CI: 1.38-3.87; $\mathrm{I}^{2}=13.6 \%$; Figure 3), especially for UC patients (SIR: 3.01 ; 95\% CI: $1.75-4.27 ; \mathrm{I}^{2}=0.0 \%$; Figure 3).

Great improvement has been made in understanding the interaction between inflammation and carcinogenesis. Current knowledge about the mechanism underlying inflammation related carcinogenesis mainly depicts inflammation induced epigenetic alterations and DNA damage as the key factors in carcinogenesis (33). IBD related malignancy has been the hot point for researchers in the past decades and agreement has been achieved on the increased risk of IBD related colorectal cancer (34). As discussed above, it is widely accepted that the chronic local inflammation in bowel is involved in the carcinogenesis of colorectal cancer (35). For extraintestinal cancers, previous epidemiological studies revealed a tight association between IBD and increased risk of numerous cancers like hematologic cancer, lung cancer and non-Hodgkin lymphoma (26,36). However, studies about the association between IBD and lower urinary tract cancers including 


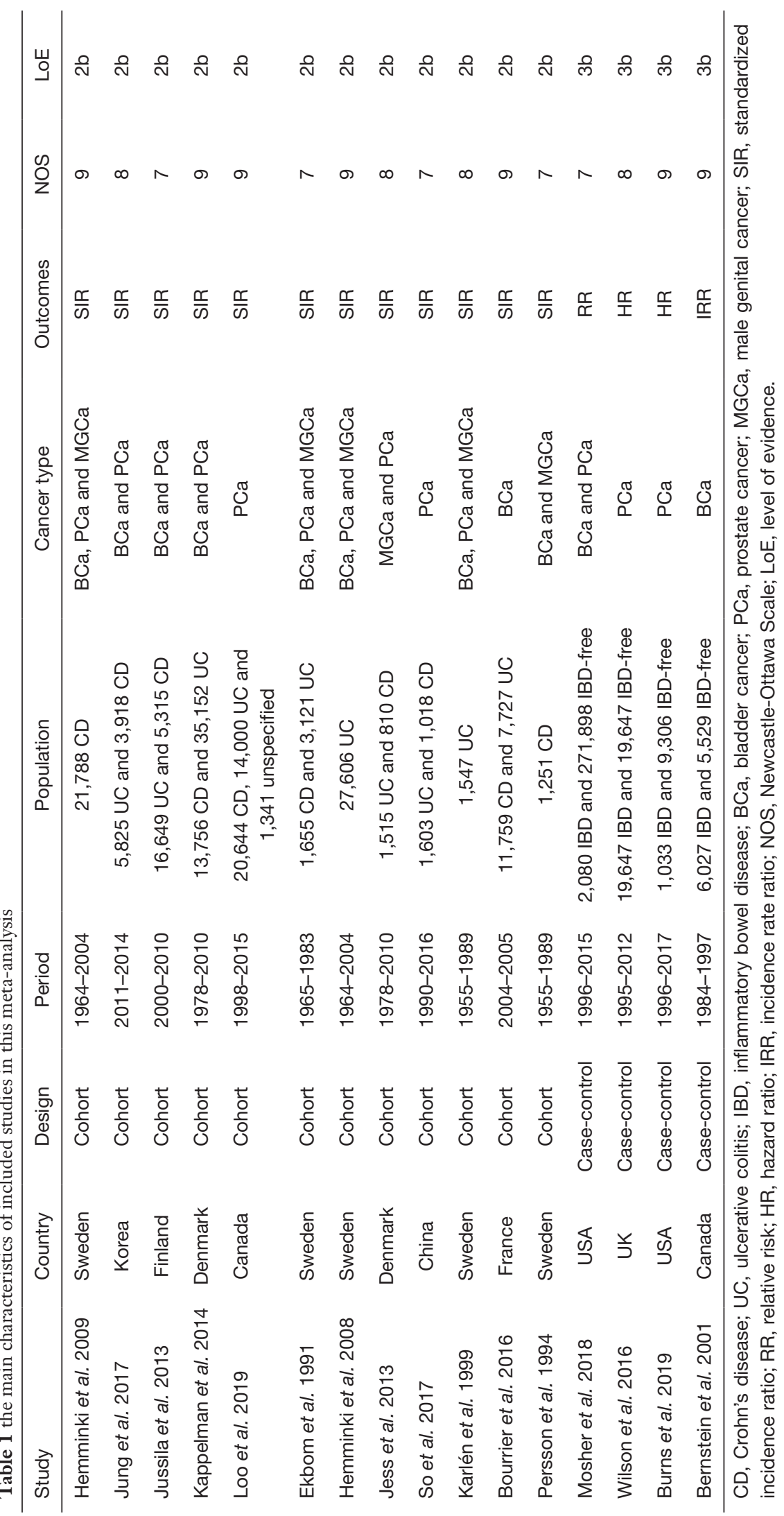


Prostate cancer

SIR

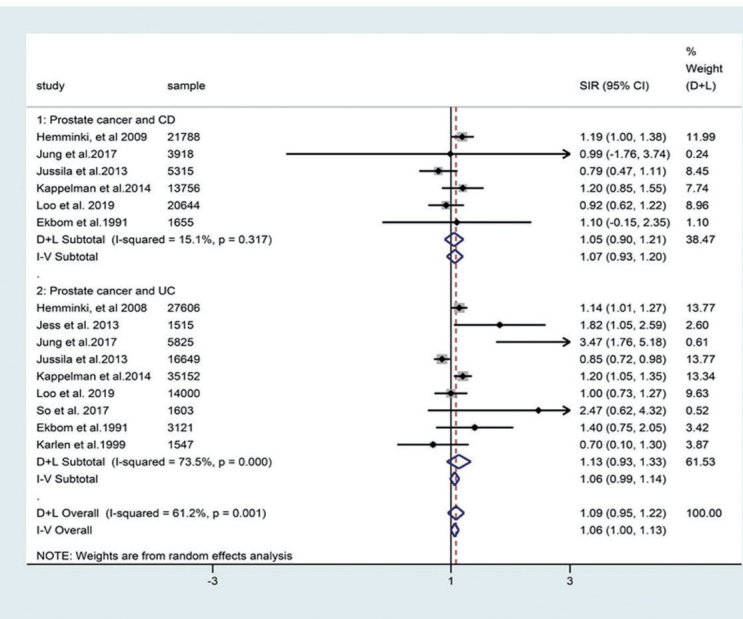

Bladder cancer

SIR

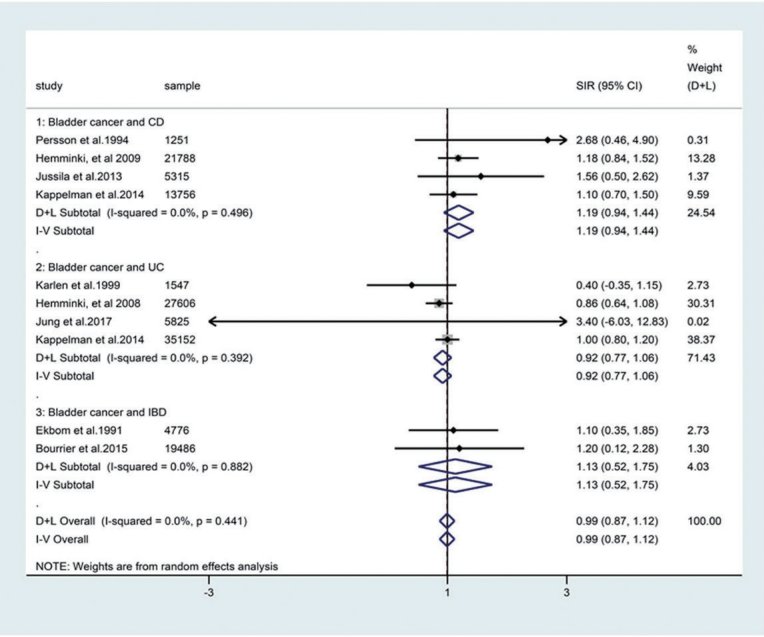

Male genital cancer

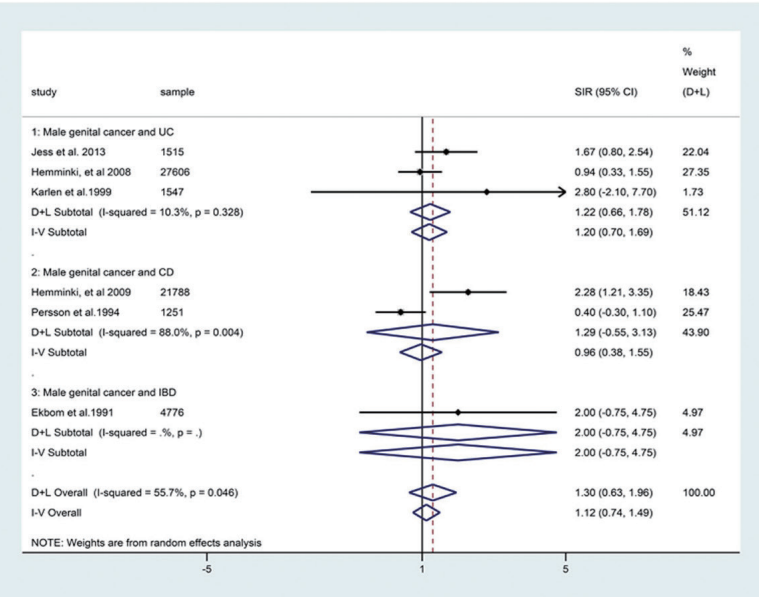

$\mathrm{RR}$

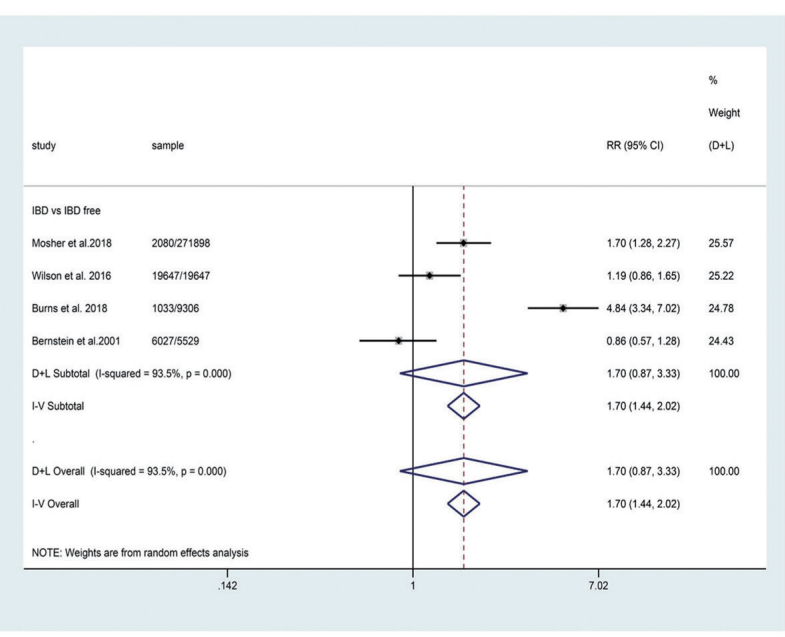

$\mathrm{RR}$

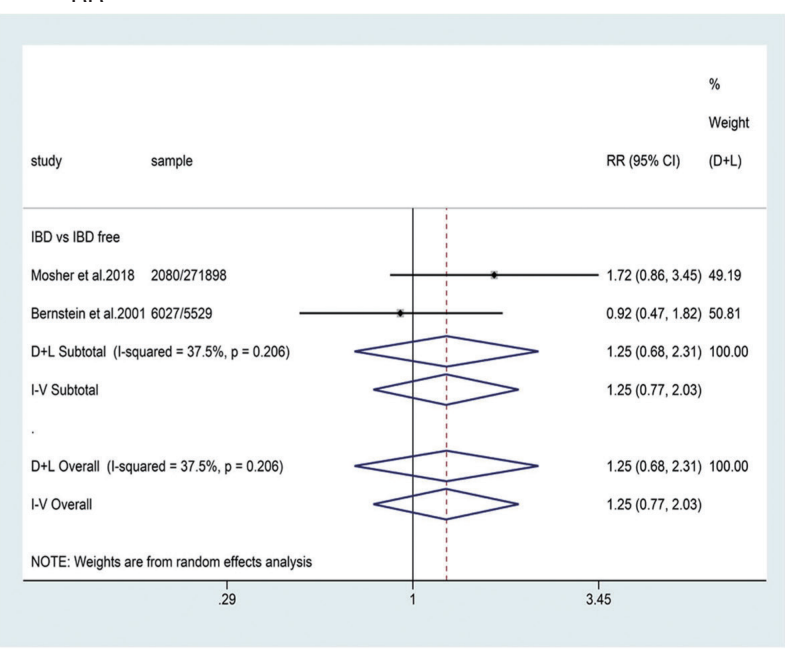

Figure 2 The pooled results of this meta-analysis. IBD, inflammatory bowel disease; UC, ulcerative colitis; CD, Crohn's disease; SIR, standardized incidence ratio; RR, relative risk; CI, confidence interval; D+L, DerSimonian-Laird; I-V, inverse-variance. 


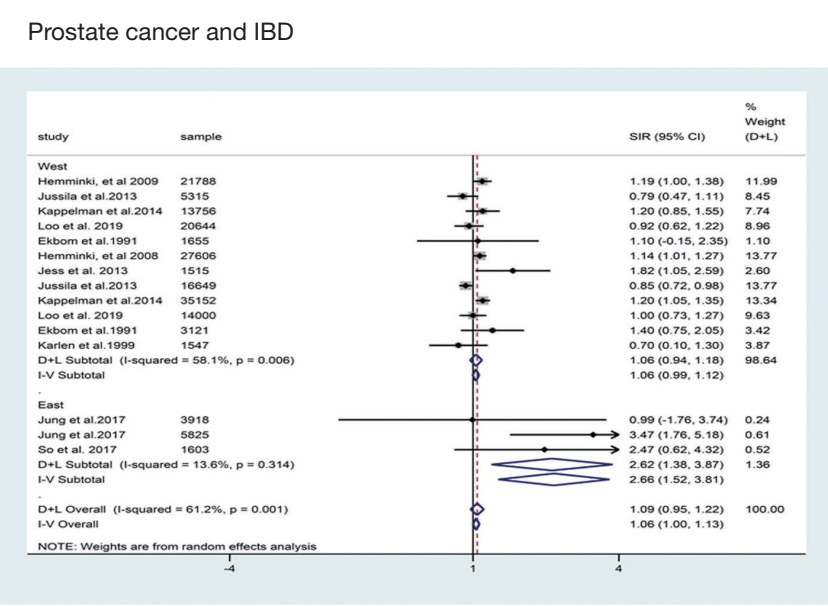

Prostate cancer and UC

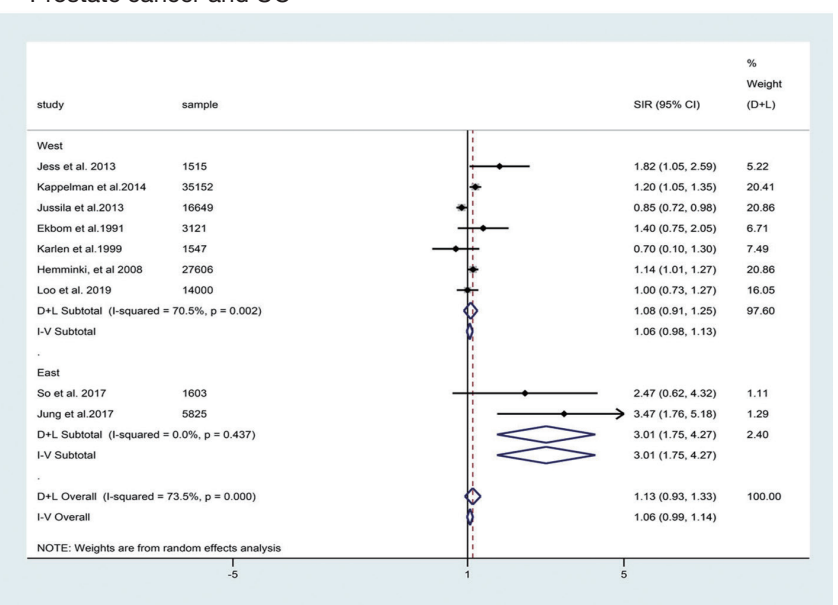

Prostate cancer and CD

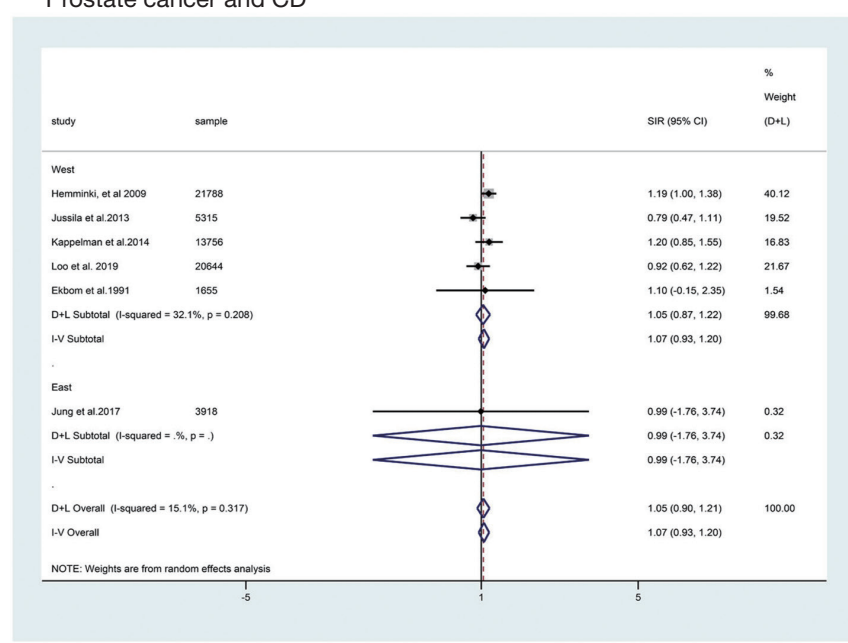

Figure 3 The funnel plots and sensitivity analyses of outcomes in this meta-analysis. IBD, inflammatory bowel disease; UC, ulcerative colitis; CD, Crohn's disease; SIR, standardized incidence ratio; CI, confidence interval; D+L, DerSimonian-Laird; I-V, inverse-variance.
BCa and MGCa are limited and no conclusive results are available. A previous meta-analysis evaluating the risk of extraintestinal cancers in IBD patients reported an increased risk of $\mathrm{BCa}$ (6). However, only 2 eligible studies were included in their pool analysis for risk of $\mathrm{BCa}$, which could weaken the strength of their conclusion. For the risk of PCa in IBD patients, controversies still remain. Some studies reported that there was an association between increased risk of PCa and IBD $(23,26,31)$. In contrast, in a previous meta-analysis of population-based studies, they found there was no increased incidence of PCa among IBD patients (6).

Though mechanism underlying increased risk of $\mathrm{PCa}$ among IBD patients in East Asian countries remains elusive, the findings of our study could still be of value in providing guidance for PCa surveillance in IBD patients, especially for those in East Asian countries. Meanwhile, as the risk of BCa was not statistically different between IBD patients and background population, we detected from the results a trend toward higher risk in IBD group. We believed that this might be due to the lack of data from Eastern countries in the included studies. These findings could be inconsistent with previous meta-analysis (6) and more qualitative studies are needed to yield a conclusive result in the future. Besides, although chronic inflammation was thought to be an important risk factor for extraintestinal cancer in IBD patients, mechanism for IBD related colorectal cancer and extraintestinal cancer might be different. Instead, immunosuppression medications that are widely used for IBD treatment have been demonstrated to be related to increased risk of numerous malignancies including non-Hodgkin lymphoma, acute myeloid leukemia and myelodysplastic syndromes, non-melanoma skin cancers and urinary tract cancers (9-12). Concerned the large range of immunosuppression agents currently available for IBD treatment and insufficient data from studies evaluating their effect on carcinogenesis in IBD patients, we were unable to perform a subgroup analysis to evaluate the effect of immunosuppression agents on the risk of cancer in IBD. Future studies conducted on this topic could be of great value in providing guidance in immunosuppression agent option for IBD treatment.

\section{Conclusions}

IBD did not significantly increase the risk of $\mathrm{PCa}, \mathrm{BCa}$ and MGCa. CD patients seemed to have a higher risk of PCa and $\mathrm{BCa}$, and $\mathrm{UC}$ patients seemed to have a higher risk of PCa. However, IBD patients, especially for UC patients, in 
Prostate cancer Funnel plot

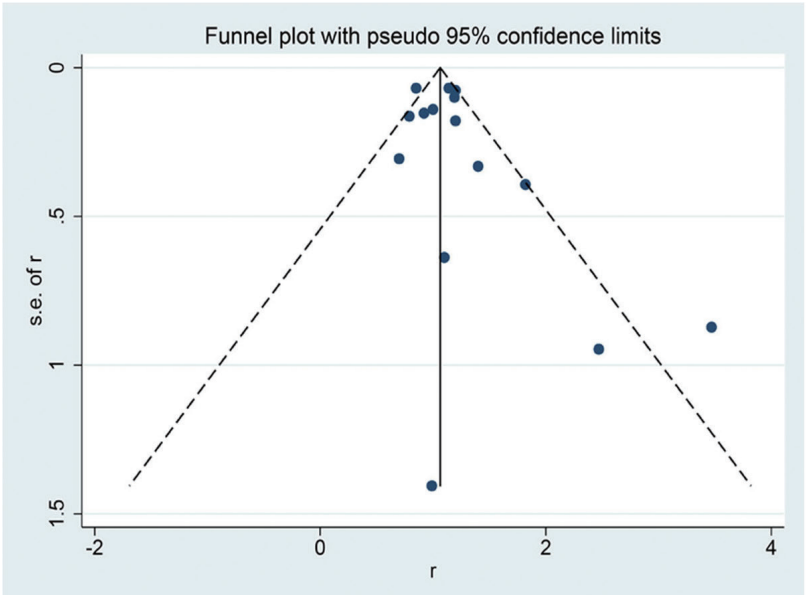

Bladder cancer

Funnel plot

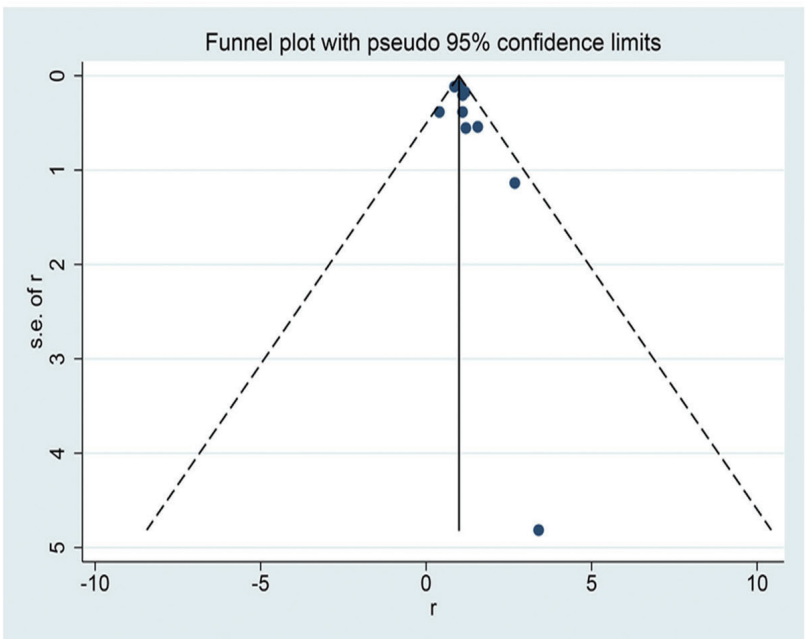

Male genital cancer

Funnel plot

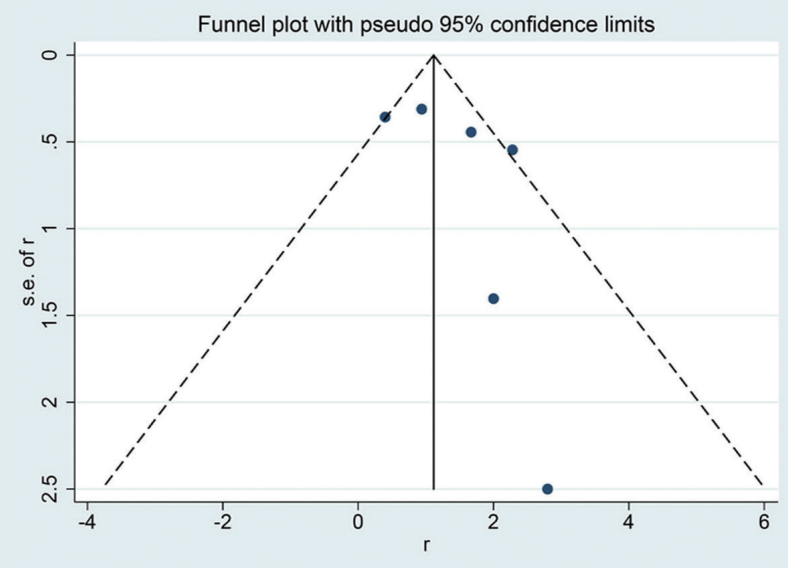

Sensitivity analysis

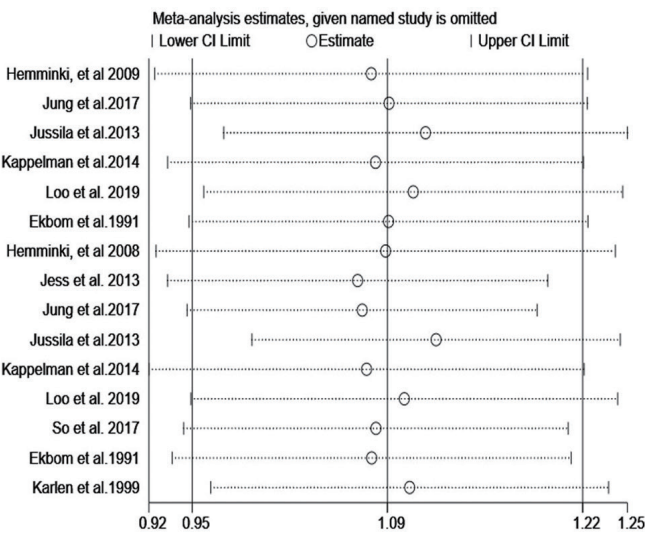

Sensitivity analysis

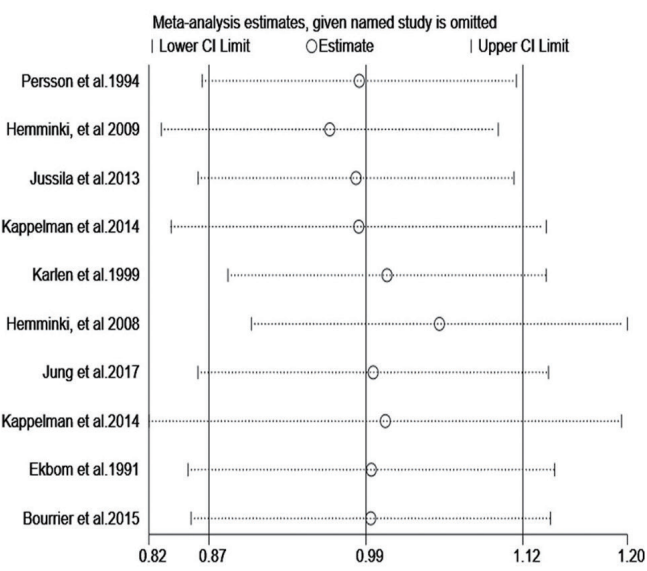

Sensitivity analysis

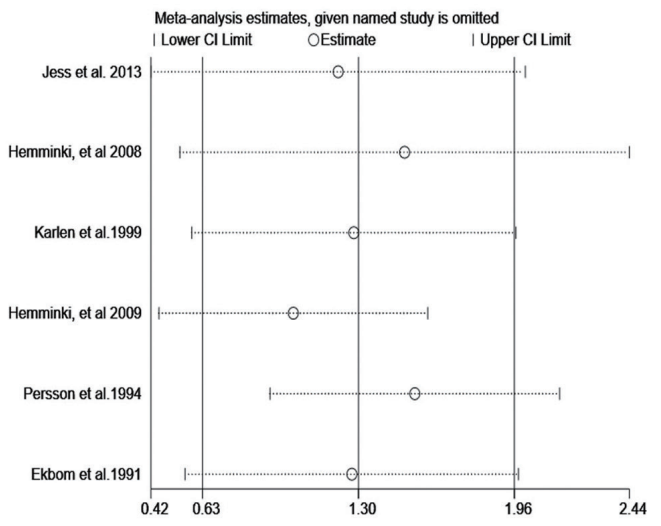

Figure 4 The subgroup analysis of prostate cancer according to geographical region. CI, confidence interval. 
Eastern countries have significantly increased PCa risk.

\section{Acknowledgments}

Funding: None.

\section{Footnote}

Reporting Checklist: The authors have completed the PRISMA reporting checklist. Available at http://dx.doi. org/10.21037/tau-20-1020

Conflicts of Interest: All authors have completed the ICMJE uniform disclosure form (available at http://dx.doi. org/10.21037/tau-20-1020). The authors have no conflicts of interest to declare.

Ethical Statement: The authors are accountable for all aspects of the work in ensuring that questions related to the accuracy or integrity of any part of the work are appropriately investigated and resolved.

Open Access Statement: This is an Open Access article distributed in accordance with the Creative Commons Attribution-NonCommercial-NoDerivs 4.0 International License (CC BY-NC-ND 4.0), which permits the noncommercial replication and distribution of the article with the strict proviso that no changes or edits are made and the original work is properly cited (including links to both the formal publication through the relevant DOI and the license). See: https://creativecommons.org/licenses/by-nc-nd/4.0/.

\section{References}

1. Chi KR. Epidemiology: Rising in the East. Nature 2016;540:S100-2.

2. Canavan C, Abrams KR, Mayberry J. Meta-analysis: colorectal and small bowel cancer risk in patients with Crohn's disease. Aliment Pharmacol Ther 2006;23:1097-104.

3. Eaden JA, Abrams KR, Mayberry JF. The risk of colorectal cancer in ulcerative colitis: a meta-analysis. Gut 2001;48:526-35.

4. Jess T, Simonsen J, Jørgensen KT, et al. Decreasing risk of colorectal cancer in patients with inflammatory bowel disease over 30 years. Gastroenterology 2012;143: 375-81. e1; quiz e13-4.

5. Yadav S, Singh S, Harmsen WS, et al. Effect of
Medications on Risk of Cancer in Patients with Inflammatory Bowel Diseases: A Population-Based Cohort Study from Olmsted County, Minnesota. Mayo Clin Proc 2015;90:738-46.

6. Pedersen N, Duricova D, Elkjaer M, et al. Risk of extraintestinal cancer in inflammatory bowel disease: metaanalysis of population-based cohort studies. Am J Gastroenterol 2010;105:1480-7.

7. Trikha M, Corringham R, Klein B, et al. Targeted antiinterleukin-6 monoclonal antibody therapy for cancer: a review of the rationale and clinical evidence. Clin Cancer Res 2003;9:4653-65.

8. Gakis $\mathrm{G}$. The role of inflammation in bladder cancer. Adv Exp Med Biol 2014;816:183-96.

9. Long MD, Herfarth HH, Pipkin CA, et al. Increased risk for non-melanoma skin cancer in patients with inflammatory bowel disease. Clin Gastroenterol Hepatol 2010;8:268-74.

10. Lopez A, Mounier M, Bouvier AM, et al. Increased risk of acute myeloid leukemias and myelodysplastic syndromes in patients who received thiopurine treatment for inflammatory bowel disease. Clin Gastroenterol Hepatol 2014;12:1324-9.

11. Beaugerie L, Brousse N, Bouvier AM, et al. Lymphoproliferative disorders in patients receiving thiopurines for inflammatory bowel disease: a prospective observational cohort study. Lancet 2009;374:1617-25.

12. Bourrier A, Carrat F, Colombel JF, et al. Excess risk of urinary tract cancers in patients receiving thiopurines for inflammatory bowel disease: a prospective observational cohort study. Aliment Pharmacol Ther 2016;43:252-61.

13. Ge Y, Shi Q, Yao W, et al. The association between inflammatory bowel disease and prostate cancer risk: a meta-analysis. Prostate Cancer Prostatic Dis 2020;23:53-8.

14. Moher D, Liberati A, Tetzlaff J, et al. Preferred reporting items for systematic reviews and meta-analyses: the PRISMA statement. PLoS Med 2009;6:e1000097.

15. Wells GA, Shea B, O'Connell D, et al. The NewcastleOttawa Scale (NOS) for assessing the quality if nonrandomized studies in meta-analyses. Ottawa Hospital Research Institute. Available online: http://www.ohri.ca/ programs/clinical_epidemiology/oxford.asp

16. Centre for Evidence-Based Medicine. Oxford Centre for evidence-based medicine: levels of evidence, 2009. Available online: http://www.cebm.net/oxfordcentreevidence-based-medicine-levels-evidencemarch-2009/

17. Persson PG, Karlén P, Bernell O, et al. Crohn's disease and 
cancer: a population-based cohort study. Gastroenterology 1994;107:1675-9.

18. Hemminki K, Li X, Sundquist J, et al. Cancer risks in Crohn disease patients. Ann Oncol 2009;20:574-80.

19. Jussila A, Virta LJ, Pukkala E, et al. Malignancies in patients with inflammatory bowel disease: a nationwide register study in Finland. Scand J Gastroenterol 2013;48:1405-13.

20. Kappelman MD, Farkas DK, Long MD, et al. Risk of cancer in patients with inflammatory bowel diseases: a nationwide population-based cohort study with 30 years of follow-up evaluation. Clin Gastroenterol Hepatol 2014;12:265-73.e1.

21. Karlén P, Löfberg R, Broström O, et al. Increased risk of cancer in ulcerative colitis: a population-based cohort study. Am J Gastroenterol 1999;94:1047-52.

22. Hemminki K, Li X, Sundquist J, et al. Cancer risks in ulcerative colitis patients. Int J Cancer 2008;123:1417-21.

23. Jung YS, Han M, Park S, et al. Cancer Risk in the Early Stages of Inflammatory Bowel Disease in Korean Patients: A Nationwide Population-based Study. J Crohns Colitis 2017;11:954-62.

24. Ekbom A, Helmick C, Zack M, et al. Extracolonic malignancies in inflammatory bowel disease. Cancer 1991;67:2015-9.

25. Loo SY, Vutcovici M, Bitton A, et al. Risk of Malignant Cancers in Inflammatory Bowel Disease. J Crohns Colitis 2019;13:1302-10.

26. Jess T, Horváth-Puhó E, Fallingborg J, et al. Cancer risk in inflammatory bowel disease according to patient phenotype and treatment: a Danish population-based cohort study. Am J Gastroenterol 2013;108:1869-76.

27. So J, Tang W, Leung WK, et al. Cancer Risk in 2621 Chinese Patients with Inflammatory Bowel Disease: A

Cite this article as: Zhang C, Liu S, Peng L, Wu J, Zeng X, Lu Y, Shen H, Luo D. Does inflammatory bowel disease increase the risk of lower urinary tract tumors: a meta-analysis. Transl Androl Urol 2021;10(1):164-173. doi: 10.21037/tau-201020
Population-based Cohort Study. Inflamm Bowel Dis 2017;23:2061-8.

28. Mosher CA, Brown GR, Weideman RA, et al. Incidence of Colorectal Cancer and Extracolonic Cancers in Veteran Patients with Inflammatory Bowel Disease. Inflamm Bowel Dis 2018;24:617-23.

29. Bernstein CN, Blanchard JF, Kliewer E, et al. Cancer risk in patients with inflammatory bowel disease: a populationbased study. Cancer 2001;91:854-62.

30. Wilson JC, Furlano RI, Jick SS, et al. A populationbased study examining the risk of malignancy in patients diagnosed with inflammatory bowel disease. J Gastroenterol 2016;51:1050-62.

31. Burns JA, Weiner AB, Catalona WJ, et al. Inflammatory Bowel Disease and the Risk of Prostate Cancer. Eur Urol 2019;75:846-52.

32. Xu J, Goodman M, Jemal A, et al. Prostate Cancer Prognostic Factors Among Asian Patients Born in the US Compared to Those Born Abroad. J Immigr Minor Health 2015;17:625-31.

33. Murata M. Inflammation and cancer. Environ Health Prev Med 2018;23:50.

34. Herrinton LJ, Liu L, Levin TR, et al. Incidence and mortality of colorectal adenocarcinoma in persons with inflammatory bowel disease from 1998 to 2010. Gastroenterology 2012;143:382-9.

35. Dulai PS, Sandborn WJ, Gupta S. Colorectal Cancer and Dysplasia in Inflammatory Bowel Disease: A Review of Disease Epidemiology, Pathophysiology, and Management. Cancer Prev Res (Phila) 2016;9:887-94.

36. van den Heuvel TRA, Wintjens DSJ, Jeuring SFG, et al. Inflammatory bowel disease, cancer and medication: Cancer risk in the Dutch population-based IBDSL cohort. Int J Cancer 2016;139:1270-80. 


\section{Inflammatory bowel diseases and bladder cancer}

\section{PubMed}

((((Inflammatory Bowel Diseases[Title/Abstract]) OR Inflammatory Bowel Disease[Title/Abstract]) OR Bowel Diseases, Inflammatory[Title/Abstract])) AND $(((()((()(((()(($ Urinary Bladder Neoplasms[Title/Abstract]) OR Neoplasm, Urinary Bladder[Title/Abstract]) OR Urinary Bladder Neoplasm[Title/Abstract]) OR Neoplasms, Bladder[Title/Abstract]) OR Bladder Neoplasms[Title/Abstract]) OR Bladder Neoplasm[Title/Abstract]) OR Neoplasm, Bladder[Title/Abstract]) OR Bladder Tumors[Title/Abstract]) OR Bladder Tumor[Title/Abstract]) OR Tumor, Bladder[Title/Abstract]) OR Tumors, Bladder[Title/Abstract]) OR Urinary Bladder Cancer[Title/Abstract]) OR Cancer, Urinary Bladder[Title/Abstract]) OR Malignant Tumor of Urinary Bladder[Title/Abstract]) OR Cancer of the Bladder[Title/Abstract]) OR Bladder Cancer[Title/ Abstract]) OR Bladder Cancers[Title/Abstract]) OR Cancer, Bladder[Title/Abstract]) OR Cancer of Bladder[Title/Abstract]) 44 records

\section{Web of Science}

\#1 TOPIC: (Inflammatory Bowel Diseases) OR TOPIC: (Inflammatory Bowel Disease) OR TOPIC: (Bowel Diseases, Inflammatory)

\#2 TOPIC: (Urinary Bladder Neoplasms) OR TOPIC: (Neoplasm, Urinary Bladder) OR TOPIC: (Urinary Bladder Neoplasm) OR TOPIC: (Neoplasms, Bladder) OR TOPIC: (Bladder Neoplasms) OR TOPIC: (Bladder Neoplasm) OR TOPIC: (Neoplasm, Bladder) OR TOPIC: (Bladder Tumors) OR TOPIC: (Bladder Tumor) OR TOPIC: (Tumor, Bladder) OR TOPIC: (Tumors, Bladder) OR TOPIC: (Urinary Bladder Cancer) OR TOPIC: (Cancer, Urinary Bladder) OR TOPIC: (Malignant Tumor of Urinary Bladder) OR TOPIC: (Cancer of the Bladder) OR TOPIC: (Bladder Cancer) OR TOPIC: (Bladder Cancers) OR TOPIC: (Cancer, Bladder) OR TOPIC: (Cancer of Bladder)

\#3 \#1 and \#2 115 records

\section{OVID}

\#1 (Inflammatory Bowel Diseases or Inflammatory Bowel Disease or Bowel Diseases, Inflammatory).kw. \#2 (Urinary Bladder Neoplasms or Neoplasm, Urinary Bladder or Urinary Bladder Neoplasm or Neoplasms, Bladder or Bladder Neoplasms or Bladder Neoplasm or Neoplasm, Bladder or Bladder Tumors or Bladder Tumor or Tumor, Bladder or Tumors, Bladder or Urinary Bladder Cancer or Cancer, Urinary Bladder or Malignant Tumor of Urinary Bladder or Cancer of the Bladder or Bladder Cancer or Bladder Cancers or Cancer, Bladder or Cancer of Bladder).kw.

$\# 3 \# 1$ and \#2 3 records

\section{Inflammatory bowel diseases and prostate cancer}

\section{PubMed}

)((((()((()((()((Prostatic Neoplasms[Title/Abstract]) OR Prostate Neoplasms[Title/Abstract]) OR Neoplasms, Prostate[Title/ Abstract]) OR Neoplasm, Prostate[Title/Abstract]) OR Prostate Neoplasm[Title/Abstract]) OR Neoplasms, Prostatic[Title/ Abstract]) OR Neoplasm, Prostatic[Title/Abstract]) OR Prostatic Neoplasm[Title/Abstract]) OR Prostate Cancer[Title/ Abstract]) OR Cancer, Prostate[Title/Abstract]) OR Cancers, Prostate[Title/Abstract]) OR Prostate Cancers[Title/Abstract]) OR Cancer of the Prostate[Title/Abstract]) OR Prostatic Cancer[Title/Abstract]) OR Cancer, Prostatic[Title/Abstract]) OR Cancers, Prostatic[Title/Abstract]) OR Prostatic Cancers[Title/Abstract]) OR Cancer of Prostate[Title/Abstract])) AND (((Inflammatory Bowel Diseases[Title/Abstract]) OR Inflammatory Bowel Disease[Title/Abstract]) OR Bowel Diseases, Inflammatory[Title/Abstract]) 109 records 


\section{Web of Science}

TOPIC: $((((()((((()((()(($ Prostatic Neoplasms) OR Prostate Neoplasms) OR Neoplasms, Prostate) OR Neoplasm, Prostate) OR Prostate Neoplasm) OR Neoplasms, Prostatic) OR Neoplasm, Prostatic) OR Prostatic Neoplasm) OR Prostate Cancer) OR Cancer, Prostate) OR Cancers, Prostate) OR Prostate Cancers) OR Cancer of the Prostate) OR Prostatic Cancer) OR Cancer, Prostatic) OR Cancers, Prostatic) OR Prostatic Cancers) OR Cancer of Prostate)) AND (((Inflammatory Bowel Diseases) OR Inflammatory Bowel Disease) OR Bowel Diseases, Inflammatory)) 305 records

\section{OVID}

\#1 (Prostatic Neoplasms or Prostate Neoplasms or Neoplasms, Prostate or Neoplasm, Prostate or Prostate Neoplasm or Neoplasms, Prostatic or Neoplasm, Prostatic or Prostatic Neoplasm or Prostate Cancer or Cancer, Prostate or Cancers, Prostate or Prostate Cancers or Cancer of the Prostate or Prostatic Cancer or Cancer, Prostatic or Cancers, Prostatic or Prostatic Cancers or Cancer of Prostate).kw.

\#2 (Inflammatory Bowel Diseases or Inflammatory Bowel Disease or Bowel Diseases, Inflammatory).kw.

\#3 1 and 222 records

\section{Inflammatory bowel disease and male genital cancer}

\section{PubMed}

((((((Genital Neoplasms, Male[Title/Abstract]) OR Neoplasms, Male Genital[Title/Abstract]) OR Male Genital Neoplasms[Title/Abstract]) OR Genital Neoplasm, Male[Title/Abstract]) OR Male Genital Neoplasm[Title/Abstract]) OR Neoplasm, Male Genital[Title/Abstract])) AND (((Inflammatory Bowel Diseases[Title/Abstract]) OR Inflammatory Bowel Disease[Title/Abstract]) OR Bowel Diseases, Inflammatory[Title/Abstract]) 3 records

Web

(((((((Genital Neoplasms, Male) OR Neoplasms, Male Genital) OR Male Genital Neoplasms) OR Genital Neoplasm, Male) OR Male Genital Neoplasm) OR Neoplasm, Male Genital)) AND (((Inflammatory Bowel Diseases) OR Inflammatory Bowel Disease) OR Bowel Diseases, Inflammatory)) 1 records

OVID

0 records 